$11 \mid 2010$

NOVECENTO... E DINTORNI

Littérature et nouveaux mass médias

\title{
Riviste letterarie on line
}

\section{Franco Manai}

\section{OpenEdition}

\section{Journals}

Edizione digitale

URL: http://journals.openedition.org/cei/122

DOI: $10.4000 /$ cei. 122

ISSN: 2260-779X

\section{Editore}

UGA Éditions/Université Grenoble Alpes

\section{Edizione cartacea}

Data di pubblicazione: 15 juin 2010

Paginazione: 103-111

ISBN: 978-2-84310-168-7

ISSN: 1770-9571

Notizia bibliografica digitale

Franco Manai, «Riviste letterarie on line», Cahiers d'études italiennes [Online], 11 | 2010, online dal 15 décembre 2011, consultato il 26 mars 2021. URL: http://journals.openedition.org/cei/122 ; DOI: https://doi.org/10.4000/cei.122 


\title{
RIVISTE LETTERARIE ON LINE
}

\author{
Franco Manai \\ Université d'Auckland
}

L'8 settembre 2007 si è celebrato a Bologna il Vaffa Day, promosso dall'attore buffo e entertainer Beppe Grillo. Circa 50 mila persone hanno riempito la grande piazza Maggiore (secondo le stime della Repubblica, ma Grillo dice che erano 200 mila), in risposta all'appello che l'attore aveva lanciato attraverso il suo blog, e hanno insieme gridato "Vaffanculo» ai politici italiani, accusati di essere una casta interessata esclusivamente al proprio vantaggio, senza relazione con i reali problemi del Paese, senza ideali, senza capacità. Più ancora del segnale di sfiducia nei confronti della classe dirigente, ciò che ha destato sensazione è stato il fatto che Grillo sia stato in grado di raggiungere un numero di persone così grande muovendosi praticamente da solo, e facendo leva su uno strumento semplice ed economico come internet, appunto tramite il suo blog.

Al di là degli esiti contingenti dell'antipolitica grillesca, ciò che emerge con prepotenza è, ancora una volta, il sogno di una cosa, il desiderio di esserci e di partecipare, il mito di una politica che coinvolge direttamente il cittadino, di un'Atene senza schiavi. Proprio questo mito, d'altronde, è quello che si è puntualmente scatenato ogni volta che la tecnica delle comunicazioni ha fatto un balzo in avanti.

In origine fu il telegrafo (I838), che separava la comunicazione dal trasporto, dallo spostamento fisico del messaggero. Il messaggio, liberato dalla persona che materialmente lo reca, assume una sua identità, un'essenza assolutamente nuova, che prepara la mente dell'uomo e della donna (inizialmente di quelli e quelle occidentali) alle successive evoluzioni. E queste puntualmente arrivano, prima col cinema (1893, col quale nasce la cultura popolare come la si intende ancora oggi), poi a ruota con la radio (I892 con Herz, ma I895 quella senza fili di Marconi, che spalanca le porte alla moderna propaganda, ma anche alla diffusione dell'informazione su una scala fino allora impensabile), quindi con la televisione, dominatrice 
incontrastata della seconda metà del xx secolo, e infine con internet, la portata del quale è ancora tutta da misurare.

Ognuna di queste svolte ha comportato, specie agli albori del nuovo mezzo, una fiducia quasi cieca nelle potenzialità che si aprivano per una vita più piena e più felice, in una società che si offriva speranzosa ai miracoli promessi da uno scambio di conoscenze sempre meno mediato e difficoltoso, sempre più accessibile ed efficiente, sempre più completo. Sappiamo quanto il sogno della trasparenza fra gli individui, e tra il singolo e il corpo sociale, sia una forza motrice della storia e della politica, e quanto gli uomini possano ostinarsi nel suo perseguimento, a onta non solo di ogni fallimento, ma anche di ogni effetto boomerang conseguito nel cercare di raggiungere quel fine.

L'avvento di internet ha aperto la strada alle speranze più ardite e ai desideri più temerari. La struttura stessa della rete sembrava garantire la più completa libertà e autonomia nell'uso del mezzo, al di là di ogni possibile condizionamento. La realtà degli ultimi anni si è fatta carico di dimostrare quanto, anche in questo caso, tali chimere fossero esageratamente fiduciose. Uno spazio cibernetico vasto come la Cina può tranquillamente essere oscurato per quanto riguarda tutto ciò che dispiace al regime. Lo stesso mondo occidentale, sotto le minacce che l'ideologia dominante decide di volta in volta di ingigantire alle dimensioni di allarmi apocalittici (il terrorismo, la pedofilia, fra un po' la criminalità spicciola extra e intracomunitaria oppure i writer deturpanti muri e vagoni ferroviari), si sta mostrando pronto, e tecnologicamente capace, di attuare un controllo capillare di tutto ciò che viene immesso nella rete, di chi lo utilizza e quando. In pratica, sta perdendo consistenza l'idea che la Rete renda possibile una libera comunicazione, immediata e senza filtri, tale da realizzare la trasparenza edenica di cui parla Starobinski a proposito di Rousseau. I casi dei blog privati e pubblici sono indicativi dell'indice di infantile delirio di onnipotenza che caratterizza la visione della Rete come luogo utopico dove liberare l'anima, dove esprimere il Messaggio che cambia la vita e la società.

Accanto all'uso ipercommerciale che è quello dominante della Rete, e accanto al suo succedaneo intorpidente, il baloccamento dei blog, esiste pure un'altra via, un altro modo di usare le opportunità offerte dalla tecnologia dell'informazione la più aggiornata, per gettare uno sguardo critico sulla realtà, e soprattutto per cercare di agire in essa sulla base di principi autenticamente trasparenti, il cui pregio consiste nell'offrirsi alla discussione con una franchezza che rischia lo sbaraglio. 
Vogliamo parlare del mondo delle riviste online, e in particolare delle riviste accademiche specializzate nella cultura umanistica in generale e in letteratura in particolare. La struttura accademica è molto importante, in quanto garantisce, da parte degli editori, una piena assunzione di responsabilità, e si sottrae di conseguenza all'accusa di anarchico qualunquismo che facilmente si può scagliare contro chi metta su un sito aperto a ogni contributo in presa diretta, senza il vaglio di un'intenzione, di un progetto.

Il mondo letterario italiano si è mosso leggermente in ritardo, anche in questo campo, rispetto ai pionieri di altri paesi. In compenso ha dimostrato, con un manipolo di coraggiose iniziative, di essere in grado di trovare una sua voce potente e originale.

Va sicuramente segnalata in primo luogo GriseldaOnLine, frutto della collaborazione tra il Dipartimento di Italianistica dell'Università di Bologna e la casa editrice Gedit. Diretta dall'italianista, studioso di Umanesimo e Rinascimento, Gian Mario Anselmi, ha cadenza annuale e ha iniziato le pubblicazioni nel 2002. Il progetto di GriseldaOnLine punta a coniugare la ricerca accademica nel campo umanistico, e in particolare in quello dell'italianistica, a un'azione di ampia divulgazione, mirata soprattutto al pubblico della scuola. Inoltre, esso si prefigge di avvicinare il mondo della ricerca umanistica, tradizionalmente poco incline alla tecnologia, all'utilizzo di ciò che oggi è tecnologicamente più avanzato, l'informatica. I vari numeri sono organizzati tematicamente (I, Inferni; II, L'altro; III, Il corpo; IV, Il nemico; V, Ai giovani; VI, Rifiuti scarti esuberi; VII A rovescio) e comprendono una serie di saggi dalle cui pagine è possibile passare tramite link ipertestuali, sia alla consultazione diretta di molti dei testi citati, sia a dei percorsi iconografici che giovano grandemente alla contestualizzazione e all'arricchimento del discorso storico e critico. L'attenzione è sempre vigile nei confronti del possibile utilizzo didattico del materiale offerto, con approfondimenti dal punto di vista metodologico. I lettori hanno la possibilità di interagire con gli estensori degli articoli e tra di loro, grazie a dei forum dedicati ai vari temi in discussione ed è anche possibile per ogni lettore chiedere l'istituzione di nuovi forum centrati appositamente su particolari aspetti che si ritengano degni di approfondimento e/o di dibattito. Notevole l'attenzione rivolta a temi importanti per l'attualizzazione dell'esperienza letteraria: basti per tutti ricordare il numero dedicato all'Altro.

All'estremo opposto di GriseldaOnLine dal punto di vista della volontà didattica, della divulgazione metodologica e della ricerca accademica è apparsa nel 2007 Buràn, quadrimestrale che propone racconti, articoli, saggi (ma anche altri contributi come fotografie) trovati in rete, la maggior 
parte nei blog o nelle home page degli autori, ma anche in riviste letterarie on line come quella venezuelana Letralia dove si pubblica un po' di tutto. I testi sono presentati in traduzione con link che rimandano agli originali ma senza commenti o indicazioni di lettura. I numeri sono monotematici (finora sono usciti Il lavoro, La città e Il conflitto) e sono divisi in due sezioni, "Il Materiale» e "L'Immaginario»: nella prima si trovano i testi che riguardano il tema di volta in volta scelto per la rivista (pare che il prossimo sia $\mathrm{Il}$ Cibo) mentre nella seconda si pubblicano testi che la redazione ritiene belli o ben fatti. Buràn non è sostenuta economicamente da nessuna istituzione e intende sfruttare le possibilità che la Rete offre sia di leggere, ascoltare e quindi scoprire «i mondi che vogliono raccontarsi» sia di condividere queste scoperte di testi non omologati all'informazione di massa con chiunque se ne voglia giovare.

Un'altra rivista on line legata a un'università è Le Simplegadi, diretta dall'anglista Antonella Riem Natale. Esce con cadenza annuale dal 2003 presso il server dell'Associazione Laureati in Lingue dell'Università di Udine. Ha come sottotitolo Rivista internazionale di lingue e letterature moderne e presenta numeri monotematici: I. La letteratura della globalizzazione, 2. Soggetti in movimento, ecc. Comprende scritture artistiche (racconti, poesie), manifesti di poetica, saggi, recensioni, in italiano e in inglese. La mira è la tessitura di una tela composita, un arazzo armonioso i cui fili sono le esperienze letterarie più diverse, attinte al di fuori di ogni confine, con attenzione tanto verso i saperi frutto di una tradizione ancestrale ininterrotta, quanto verso le possibilità offerte dalle più moderne tecnologie. Il leitmotiv è la necessità di un'apertura autentica verso gli altri per poter essere davvero aperti verso se stessi, nella convinzione che solo nel movimento di reciproca accettazione e conoscenza sia possibile e produttivo costruire un mondo pieno di senso e gravido di futuro.

El Ghibli rivista on line della letteratura della migrazione esce dal 2003 pubblicata dalla Provincia di Bologna. Trimestrale, diretta dallo scrittore migrante Pap Khouma: è la rivista della scrittura migrante per eccellenza, a cominciare dalla sua redazione, composta quasi interamente da scrittori immigrati in Italia, da un periodo più o meno lungo. Si articola in quattro sezioni principali: Racconti e poesie, per gli scrittori migranti in Italia, che usano l'italiano come lingua d'espressione letteraria; Parole dal mondo, per gli scrittori migranti non italiani nel mondo; Stanza degli ospiti, un tributo di ospitalità agli scrittori stanziali italiani e stranieri - i viaggiatori immobili - con cui è sempre più necessario interagire e collaborare per un arricchimento reciproco; Generazione che sale, dedicata a bambini e ragazzi, italiani e migranti. Non manca uno spazio di approfondimento 
critico, fornito dalla sezione delle Recensioni, ovviamente dedicata alle scritture che più direttamente hanno attinenza con il tema della migrazione delle culture, delle mescidanze e contaminazioni tra popoli e razze.

Sagarana nasce nel 2000 come parte di un progetto più vasto cresciuto attorno all'omonima scuola di scrittura di stanza a Pistoia. È divisa in sezioni 'tradizionali' come narrativa, saggi e poesie a cui sono affiancate sezioni quali Spazio Sagarana che accoglie le opere migliori della Scuola di Master Sagarana assieme a opere di autori già noti, e lo Spazio Lettori riservato appunto agli scritti inviati dai lettori. Ci sono anche altre rubriche, quali quella dei Dicas e cioè dei «consigli, dritte ai lettori». La rivista on line mira in primo luogo a offrire una piattaforma pubblica ai migliori studenti della scuola. Allo stesso tempo, però, intende anche svolgere un'azione di promozione e diffusione della conoscenza di opere in prosa e in versi, sia di autori famosi sia di autori sconosciuti, allo scopo di mantenere viva presso un pubblico che si augura sempre più vasto la conoscenza di quella che gli editori della rivista considerano la vera letteratura. Data anche la personalità del direttore Julio Monteiro Martins, scrittore migrante brasiliano, la rivista si focalizza su tematiche di forte impegno politico libertario e sulla valorizzazione delle opere di scrittura migrante. In Sagarana lo studio della letteratura è vissuto come attività creativa, che attraverso una strumentazione scientifica che abbraccia tanto la storia politica quanto la sociologia dei costumi insieme alla psicologia e alle tecniche retoriche, mira a ciò che, per esempio, in un paese laboratorio come il Sud Africa si chiamerebbe empowerment: un complesso di azioni e iniziative volte a conferire a chi non l'ha mai avuto il potere di esprimersi liberamente, di agire consapevolmente, di vivere pienamente la propria vita.

Sia Le Simplegadi che El Ghibli e Sagarana si distinguono per un'attenzione estrema verso le culture altre, in uno strenuo impegno verso uno svecchiamento della cultura e della letteratura italiane visto come urgentissimo e improcrastinabile. Possiamo forse rintracciare, all'interno del panorama culturale e accademico italiano, un ispiratore importante del lavoro e dell'orientamento di queste riviste nella figura di Armando Gnisci, comparatista della Sapienza di Roma e direttore di un'altra rivista on line, Kúmá.

A partire dai primi anni novanta Gnisci si è dedicato, con sempre maggior convinzione, a un importante lavoro di ampliamento degli orizzonti culturali del nostro Paese, non però semplicemente nel senso di una generica maggiore apertura nei confronti di quanto si pensa e si produce in altre parti del mondo, magari più avanzate di noi nel campo della ricerca 
in questo o quell'ambito accademico, ma nel senso di una rivoluzione copernicana nell'atteggiamento mentale di base con cui la cultura e la letteratura vengono viste, studiate, praticate. Su questi fondamenti è prima nato Basili (1997), un database contenente la bibliografia degli scrittori migranti in italiano, cioè degli scrittori di origine straniera che hanno scelto di esprimersi nella nostra lingua. In un secondo momento (200I), a una versione potenziata di Basili si è accompagnata la fondazione di Kúmá, rivista on line dedicata non solo agli stranieri che scrivono in italiano, come è principalmente il caso di El Ghibli, ma precipuamente all'elaborazione concettuale e alla realizzazione pratica dei due principi guida della decolonizzazione e della creolizzazione.

Le diverse sezioni della rivista (Narrativa, Teatro Cinema, Musica, Poesia, Critica, Poetica) abbracciano, com'è evidente dai titoli, un ampio spettro di tipologie artistiche, e affiancano la pubblicazione di testi, in prosa e in versi, agli interventi critici di stampo più propriamente saggistico e scientifico. Alle sezioni si aggiungono poi le rubriche (Novità editoriali, Decolonizziamoci, Interculturalità, Iniziative, Strumenti, Links, Bollettino Basili), che si propongono come utili strumenti di lavoro per chi voglia tenersi aggiornato sugli sviluppi di questo orientamento culturale nei suoi vari risvolti. I lettori possono anche iscriversi alla lista di posta elettronica degli Amici di Kúmá, che funziona come una sorta di bollettino attraverso il quale si è tenuti al corrente su eventi e iniziative, dall'uscita di libri, alle segnalazioni di convegni, di premi letterari, incontri, dibattiti, feste, ecc., inerenti al progetto culturale della rivista i quali spesso sfuggono all'attenzione di altre liste letterarie di posta elettronica o di altri bollettini letterari quali a esempio il pregevole e utile Bollettino 'goo.

Il progetto sotteso a Kúmá è dunque quello della decolonizzazione in vista di una possibile e auspicabile creolizzazione. Quando Gnisci parla di decolonizzazione, però, non intende semplicemente il processo di completamento della liberazione dei Paesi un tempo colonie europee, e ora afflitti dai mille mali causati dalla necessità di dover riprendere un cammino di progresso e autonomia politica dopo che il filo su cui si muovevano è stato spezzato per secoli. Secondo Gnisci, il processo della decolonizzazione, oggi, deve in primo luogo concentrarsi sui Paesi europei (e nordamericani), cioè sui Paesi che furono gli attori principali dell'oppressione colonialista e imperialista. L'agire colonialistico ha infettato, come un virus senza rimedio, le strutture stesse della civiltà europea, e se si vuole mettere un punto e a capo, e per lo meno provare a rilanciare un cammino di progresso verso un futuro meno disastroso di quanto non sia stato il passato, occorre partire proprio dalla guarigione dalla malattia che per 
tanto tempo ha segnato il Colonizzatore, ancor di più di quanto non abbia devastato il Colonizzato. Si tratta di apprendere a vedere se stessi e il resto del mondo in un'ottica libera dagli schemi che sono diventati, nel corso di tanti secoli, pericolosi automatismi. Questo processo non può essere affrontato dal Colonizzatore in solitudine: egli non saprebbe da che parte cominciare. Per poter anche solo intraprendere questo difficile cammino, è imprescindibile assicurarsi l'apporto del Colonizzato, al quale i secoli di oppressione hanno insegnato una strategia di esistenza che non poteva essere basata sul privilegio del potere, ma doveva per forza di cose fare aggio sul "fondamentalmente umano», su ciò che caratterizza l'uomo in quanto tale, al di là dei ruoli gerarchici in cui un destino capriccioso può gettarlo ad arbitrio. Su questa strada, allora, sarà forse possibile che l'Europeo muova anche qualche passo nella direzione del superamento dei rapporti bloccati uomo donna, adulto giovane/bambino, ricco povero, del superamento cioè di quelle categorizzazioni gerarchiche che tanto pesantemente condizionano l'esperienza esistenziale della civiltà occidentale. Lo sbocco di questo percorso, secondo Gnisci, non può che essere quella che lui chiama la creolizzazione dell'Europa (Creolizzare l'Europa è il sottotitolo della rivista). Gnisci si appoggia alle teorizzazioni di Edouard Glissant, per il quale:

La creolizzazione è il risultato del contatto tra culture diverse in uno spazio definito, che ha originato qualcosa di assolutamente nuovo e imprevedibile perché non legato a ciascun elemento separato che lo costituisce.

Poiché l'esito del processo in corso è, e non può che essere, imprevedibile, occorre, da parte degli attori di questo processo, ma altresì da parte di coloro che questo processo intendono seguire, studiare e, possibilmente, aiutare e guidare, occorre, dunque, adottare un atteggiamento di apertura e di disponibilità.

L'arte, la letteratura, la musica sono i luoghi in cui davvero è possibile che questo indispensabile incontro abbia luogo, gli spazi dove potrebbe riuscire la formazione di un linguaggio comune costruito insieme dal Visitatore e dal Visitato. Da qui l'importanza che Kúmá assegna alla pratica e allo studio della scrittura migrante, cioè, in senso lato, della scrittura prodotta da donne e uomini di-spiazzati, che sono usciti dalla loro piccola piazza, che si sono allontanati, per forza o per scelta, il più delle volte per forza $e$ per scelta, dal mondo sempre piccolo cui inizialmente sono appartenuti.

Gnisci è convinto che questo processo di mutua compenetrazione, di reciproca apertura, al di là degli epifenomeni di intolleranza e di incomprensione, stia comunque avendo luogo in misura e con una velocità 
molto maggiori di quanto noi siamo in grado di percepire e registrare. Il compito di una rivista come "Kúmá», allora, e di coloro che si riconoscono in questo ambizioso progetto è, come si è accennato, quello di tener d'occhio il processo in atto, accompagnarlo, aiutarlo a trovare voce e consapevolezza.

In quest'ottica, la scelta di fare una rivista online non è semplicemente legata a esigenze pratiche di risparmio di tempo e denaro, ma è piuttosto obbligata dalla volontà di assecondare l'aggancio di questo movimento con una modernità che non può essere lasciata come appannaggio delle forze della resistenza al cambiamento e dell'arroccamento vetero-imperialistico. Proprio la natura ambigua della rete, come quella di tutte le tecnologie della comunicazione succedutesi nel tempo sul palcoscenico del progresso umano, rende imprescindibile che venga operato un riscatto politico e ideologico di uno strumento troppo prezioso per essere abbandonato nelle mani di giovani che il consumismo globale ha rimbecillito fino al delitto, o di mestatori che calibrano il tempismo dei loro exploit rivoluzionari a seconda di interessi politici decisamente sospetti. Usata in maniera consapevole, la Rete può diventare il prototipo di quello spazio di incontro, di mescidanza, di creolizzazione rizomatica che, seppure ancora in larga misura utopico, non può che essere la realtà di domani.

\section{Bibliografia}

Bollettino del 'goo (1995-), <http://www3.unibo.it/boll9oo/>.

Buran (2007-), <http://www.buran.it/buran.htm>.

Basili (1997-), <http://www3.unibo.it/bollyoo/>.

Czitrom Daniel J., Media and the American Mind. From Morse to McLuhan, Chapel Hill, Univ. of North Carolina Press, 1982.

De Gregorio Concita, "Silvio, Michela e il partito dei gazebo", in La Repubblica, 20 novembre 2007.

El Ghibli (2003-), <http://www.el-ghibli.provincia.bologna.it>.

GiacCHÉ P., «Tutta la notte ci cantano i Grillo», in Lo straniero 89, <http:// www.lostraniero.net/pagine/uno.html> [consultato il 27.II.2007].

Glissant Édouard, Introduction à une poétique du divers, Paris, Gallimard, 1996.

Glissant Édouard, «Migrazioni e creolizzazione nelle Americhe», in Kúmá 6, <http://www.disp.let.uniromar.it/kuma/kuma6.html> [consultato il 27.II.2007]. 
Gnisci Armando, La Letteratura del mondo, Roma, Sovera multimedia, I993.

-, Ascesi e decolonizzazione, Roma, Lithos, 1996.

—, La letteratura italiana della migrazione, Roma, Lilith, 1998.

-, Una storia diversa, Roma, Meltemi, $200 \mathrm{I}$.

- Via della decolonizzazione europea, Isernia, Cosmo Iannone, 2004.

-, Mondializzare la mente. Via della decolonizzazione europea, Isernia, Cosmo Iannone, 2006.

Griselda (2002-), <http://www.griseldaonline.it>.

Holdom S., «E-Journal Proliferation in Emerging Economies: The Case of Latin America", in Literary and Linguistic Computing, vol. 20, n. 3 , 2005, pp. 35I-365.

Kúmá (200I-), <http://www.disp.let.uniromaI.it/kuma/kuma.html>.

Le Simplegadi (2003-), <http://web.uniud.it/all/simplegadi>.

Letralia (1996-), <http://www.letralia.com>.

Rheingold Howard, The Virtual Community: Homesteading on the Electronic Frontier, [Ied 1993], Cambridge, Mass, MIT Press, 2000.

Sagarana (2000-), <http://www.sagarana.net>.

Starobinski Jean, Jean-Jacques Rousseau. La trasparenza e l'ostacolo, [Ied. I958], Bologna, Il Mulino, 1999.

Thornton Alinta, Does Internet Create Democracy, <http://www.zip. com.au/ athornto/intro.htm>, 2002. 
